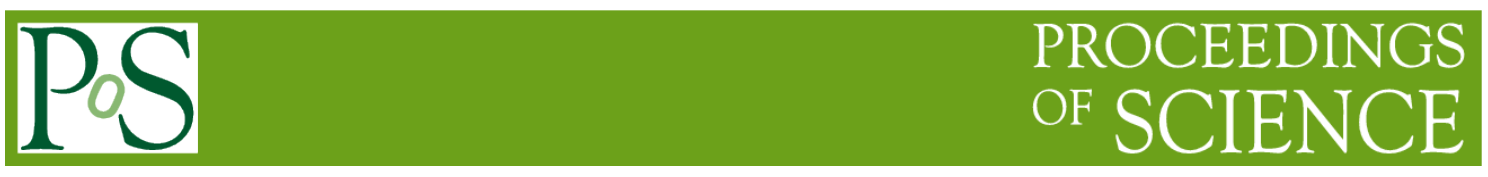

\title{
Integrated luminosity measurement at CEPC
}

\author{
I. Smiljanic ${ }^{a, *}$, I. Bozovic-Jelisavcic ${ }^{a}$, G. Kacarevic ${ }^{a}$, S. Hou ${ }^{b}$ and H. Zhu ${ }^{c}$ \\ ${ }^{a}$ Vinca Institute of Nuclear Sciences, National Institute of the Republic of Serbia, University of Belgrade, \\ M. Petrovica Alasa 12-14, Belgrade, Serbia \\ ${ }^{b}$ Academia Sinica - Institute of Physics, \\ No. 128, Section 2, Academia Rd, Nangang District, Taipei, Taiwan \\ ${ }^{c}$ Institute of High Energy Physics, CAS, \\ 19 Yuquan Rd, Shijingshan District, Beijing, China \\ E-mail: i.smiljanicevinca.rs, ibozovicevinca.rs
}

\begin{abstract}
The very forward region of a detector at future $e^{+} e^{-}$collider is the one of the most challenging regions to instrument. A luminometer - compact calorimeter dedicated for precision measurement of the integrated luminosity at a permille level or better is needed. Here we review a feasibility of such precision at CEPC, considering detector mechanical precision and beamrelated requirements. We also discuss capabilities of experimental determination of the effective centre-of-mass energy determination, from the perspective of integrated luminosity precision requirements at the $Z^{0}$ pole.
\end{abstract}

40th International Conference on High Energy physics - ICHEP2020

July 28 - August 6, 2020

Prague, Czech Republic (virtual meeting)

"Speaker 


\section{Introduction}

The Circular Electron Positron Collider (CEPC) is a large international scientific facility proposed by the Chinese particle physics community in 2012 to test the validity scale of the Standard Model (SM) in precision measurements in the Higgs, BSM and EW sector. It is designed to operate as a $\mathrm{Z}$ factory, WW and a Higgs factory [1]. In order to achieve precision required by the CEPC physics program, relative uncertainty of the integrated luminosity should be of order of $10^{-4}$ at the $Z^{0}$ pole and of order of $10^{-3}$ at $240 \mathrm{GeV}$.

\section{Systematic uncertainties of integrated luminosity from mechanics and MDI}

Systematic uncertainties from detector and machine-detector interface (MDI) related effects have been quantified through a simulation study, assuming $10^{7}$ Bhabha scattering events generated using BHLUMI [2], at $240 \mathrm{GeV}$ and $\mathrm{Z}^{0}$ pole. Luminometer axis is set at the outgoing beam, at $0.95 \mathrm{~m}$ from the interaction point (IP), with the fiducial volume acceptance between 53 mrad and 79 mrad. The crossing angle between colliding beams is set to be $33 \mathrm{mrad}$. We have applied event selection that is asymmetric in polar angle acceptance on the left and right arms of the detector, as in [3] leading to cancelation of systematic uncertainties caused by possible leftright asymmetry in an event. Comparison of results against the full fiducial volume counting is given in Table 1, at $240 \mathrm{GeV}$ center-of-mass energy and at the $Z^{0}$ pole.

Table 1: Summary of the systematic limits from mechanics and MDI in the integrated luminosity measurement at $240 \mathrm{GeV}$ and $91.2 \mathrm{GeV} \mathrm{CEPC}$, assuming $10^{-3}$ an $10^{-4}$ luminosity precision, respectively.

\begin{tabular}{|c|l|l|l|}
\hline parameter & $\begin{array}{l}\text { limit@240 GeV } \\
\text { symmetric sel. }\end{array}$ & $\begin{array}{l}\text { limit@240 GeV } \\
\text { asymmetric sel. }\end{array}$ & $\begin{array}{l}\text { Limit@91.2 GeV } \\
\text { asymmetric sel. }\end{array}$ \\
\hline$\Delta \mathbf{E C M}(\mathbf{M e V})$ & 120 & 120 & 5 \\
\hline $\mathbf{E}_{\mathrm{e}+}-\mathbf{E}_{\mathrm{e}-}(\mathbf{M e V})$ & 120 & 240 & 11 \\
\hline$\Delta \mathbf{x}_{\mathrm{IP}}(\mathbf{m m})$ & 0.1 & 1.0 & 0.5 \\
\hline$\Delta \mathbf{z}_{\mathrm{IP}}(\mathbf{m m})$ & 1.4 & 10.0 & 2.0 \\
\hline beam synch. (ps) & 1 & 15 & 3 \\
\hline$\Delta \mathbf{r}_{\text {in }}(\boldsymbol{\mu m})$ & 13 & 10 & 1 \\
\hline $\mathbf{R}_{\mathbf{r}}(\mathbf{m m})$ & 0.15 & 1.00 & 0.20 \\
\hline$\Delta \mathbf{d}(\mathbf{m m})$ & 1.00 & 1.00 & 0.08 \\
\hline$\sigma_{X_{I P}}(\mathbf{m m})$ & 0.1 & 1.0 & 0.5 \\
\hline$\sigma_{Z_{I P}}(\mathbf{m m})$ & 1 & 10 & 7 \\
\hline$\Delta \varphi(\mathbf{m r a d})$ & 6.0 & 6.0 & 0.8 \\
\hline
\end{tabular}

The MDI related effects list as follows: uncertainty of the average net center-of-mass energy $\left(\Delta \mathrm{E}_{\mathrm{CM}}\right)$, uncertainty of the asymmetry in energy of the beams, $\left(\left|\mathrm{E}_{\mathrm{e}+}-\mathrm{E}_{\mathrm{e}-}\right|\right)$, radial and axial IP position displacements $\left(\Delta \mathrm{x}_{\mathrm{IP}}, \Delta \mathrm{Z}_{\mathrm{IP}}\right)$. Considered detector-related uncertainties arising from manufacturing, positioning and alignment are: uncertainty of the luminometer inner radius $\left(\Delta \mathrm{r}_{\text {in }}\right)$, spread of the measured radial shower position w.r.t. to the true impact position $\left(\mathrm{R}_{\mathrm{r}}\right)$, uncertainty of the longitudinal distance between left and right halves of the luminometer $(\Delta \mathrm{d})$, radial and axial mechanical fluctuations of the luminometer position $\left(\sigma_{X_{\text {Ip }}}, \sigma_{Z_{\mathrm{IP}}}\right)$ and twist of the calorimeters corresponding to different rotations of the left and right detector axis $(\Delta \varphi)$. 


\section{Systematic uncertainties of integrated luminosity from the uncertainty of the effective center-of-mass energy}

As proposed for FCC-ee [4], acollinearity of muons from $e^{+} e^{-} \rightarrow \mu^{+} \mu^{-}$measured in the central tracker can be employed to determine effective center-of-mass energy $s$ ' critically limiting precision for the cross-section calculations (Table 1):

$$
\frac{s^{\prime}}{s}=\frac{\sin \theta^{+}+\sin \theta^{-}-\left|\sin \left(\theta^{+}+\theta^{-}\right)\right|}{\sin \theta^{+}+\sin \theta^{-}+\left|\sin \left(\theta^{+}+\theta^{-}\right)\right|}
$$

With WHIZARD 2.6.2 [5] we generated $250 \mathrm{~K}$ and $100 \mathrm{~K}$ di-muon events at the $Z^{0}$ pole and 240 $\mathrm{GeV}$, respectively, showing that the effective center-of-mass energy can be determined better than $10 \mathrm{MeV}$, after 2 minutes of data taking at the $\mathrm{Z}^{0}$ pole, assuming the nominal polar angle resolution of the central tracker at CEPC $(0.1 \mathrm{mrad})[1]$.

Table 2. Beam-spread variations experimentally accessible at CEPC and FCCee.

\begin{tabular}{|l|l|l|l|l|l|l|}
\hline CEPC & $\begin{array}{l}\text { Luminosity @ } \\
\mathbf{I P}\left(\mathbf{c m}^{-2} \mathbf{s}^{-1}\right)\end{array}$ & $\begin{array}{l}\text { Nominal } \\
\text { beam-spread } \\
(\%)\end{array}$ & $\begin{array}{l}\text { Number of } \\
\text { events }\end{array}$ & $\begin{array}{l}\text { Cross-section } \\
\boldsymbol{e}^{+} \boldsymbol{e}^{-} \rightarrow \boldsymbol{\mu}^{+} \boldsymbol{\mu}^{-}\end{array}$ & $\begin{array}{l}\text { Collecting } \\
\text { time }\end{array}$ & $\begin{array}{l}\text { Beam-spread } \\
\text { variation }\left(\boldsymbol{\delta} \mathbf{E}_{\mathbf{b}}\right)\end{array}$ \\
\hline $\mathbf{Z}^{\mathbf{0}}$ pole & $3.2 \cdot 10^{35}$ & 0.080 & $250 \mathrm{KEvt.}$ & $1.5 \mathrm{nb}$ & $\begin{array}{l}\sim 4 \mathrm{~min} \\
\left(2 \mathrm{~min} \text { for } 10^{-4}\right. \\
\text { of } \Delta \mathrm{L} / \mathrm{L})\end{array}$ & $\begin{array}{l}\sim 2.5 \cdot 10^{-2} \cdot \delta \mathrm{E}_{\mathrm{b}} \\
(900 \mathrm{keV})\end{array}$ \\
\hline $\mathbf{2 4 0 ~ G e V}$ & $3.0 \cdot 10^{34}$ & 0.134 & $100 \mathrm{KEvt.}$ & $4.1 \mathrm{pb}$ & $\begin{array}{l}\sim 10 \text { days } \\
\sim 0.15 \cdot \delta \mathrm{E}_{\mathrm{b}} \\
(\sim 24 \mathrm{MeV})\end{array}$ \\
\hline
\end{tabular}

\section{Discussion and summary}

We have shown that the uncertainty of the luminometer inner radius at the micron level together with the uncertainty of the available center-of-mass energy are critical requirements on the integrated luminosity measurement. Required precision of the effective center-of-mass energy can be reached from experimental determination of the muon's acolinearity after only 2 minutes of collecting di-muon events at the $Z^{0}$ pole CEPC. More details on this research can be found in [6].

\section{References}

[1] CEPC Conceptual Design Report (CDR), Volume II Physics and Detector, 2018, 381 pp, IHEP-CEPC-DR-2018-02.

[2] S. Jadach et al., Upgrade of the Monte Carlo program BHLUMI for Bhabha scattering at low angles to version 4.04, Comput. Phys. Commun. 102 (1997) 229-251.

[3] G. Abbiendi et al. [OPAL Collaboration], Precision luminosity for Z0 line shape measurements with a silicon tungsten calorimeter, Eur. Phys. J. C14 (2000) 373-425.

[4] P. Janot, Beam Energy Spread Measurement @ FCC-ee, talk given at the FCC-ee Polarization Workshop, CERN, 2017

[5] W. Kilian, T. Ohl, J. Reuter, WHIZARD: Simulating Multi-Particle Processes at LHC and ILC, Eur.Phys.J.C71 (2011) 1742, arXiv: 0708.4233 [hep-ph]

[6] I. Smiljanic, I. Bozovic Jelisavcic and G. Kacarevic, Systematic uncertainties from mechanics and MDI in luminosity measurement at CEPC, arXiv:2010.15061 [physics.insdet], 2020 